\title{
Drag Moderation by the Melting of an Ice Surface in Contact with Water
}

\author{
Ivan U. Vakarelski, ${ }^{1, *}$ Derek Y. C. Chan, ${ }^{2,3, \dagger}$ and Sigurdur T. Thoroddsen ${ }^{1}$ \\ ${ }^{1}$ Division of Physical Sciences and Engineering and Clean Combustion Research Centre, \\ King Abdullah University of Science and Technology (KAUST), Thuwal 23955-6900, Saudi Arabia \\ ${ }^{2}$ School of Mathematics and Statistics, University of Melbourne, Parkville, Victoria 3010, Australia \\ ${ }^{3}$ Department of Chemistry and Biotechnology, Swinburne University of Technology, Hawthorn, Victoria 3122, Australia
}

(Received 29 January 2015; revised manuscript received 8 June 2015; published 24 July 2015)

\begin{abstract}
We report measurements of the effects of a melting ice surface on the hydrodynamic drag of ice-shellmetal-core spheres free falling in water at a Reynolds of number $\operatorname{Re} \sim 2 \times 10^{4}-3 \times 10^{5}$ and demonstrate that the melting surface induces the early onset of the drag crisis, thus reducing the hydrodynamic drag by more than 50\%. Direct visualization of the flow pattern demonstrates the key role of surface melting. Our observations support the hypothesis that the drag reduction is due to the disturbance of the viscous boundary layer by the mass transfer from the melting ice surface.
\end{abstract}

The perception gained from common activities such as ice skating or driving on frozen wintry roads is that the characteristic lubricity of icy surfaces is due to the presence of a surface water film. The question as to why such a film exists below the melting temperature of ice has occupied great minds since the 19th century, including those of Michael Faraday, Lord Kelvin, Osborne Reynolds, and Josiah Willard Gibbs, and remains an active area of research today [1-5]. Surprisingly, in spite of the obvious relevance, for example, to the movement of icebergs $[2,6]$ in a warming climate, there had been few investigations related to friction and drag of melting ice surfaces in contact with bulk water. To the best of our knowledge, no experimental attempts had been made to quantify the hydrodynamic drag on ice-covered bluff bodies moving in water. Perhaps this is due to the intuitive presumption that the drag on icy surfaces should be no different from the drag on a solid surface. In this Letter we investigate the drag on ice-covered bodies in contact with water by studying the free fall of ice-shell-metal-core spheres in water. A comparison of varying drag reduction of ice spheres in water of different temperatures and hence melting rates supports the notion that the mass transfer and phase change at the ice-water interface provides a drag reduction mechanism that hitherto has not been considered.

The hydrodynamic drag on a smooth solid sphere of diameter $D$ moving at velocity $U$ in a fluid of density $\rho$ is traditionally characterized in terms of the drag coefficient, $C_{D}=8 F_{D} /\left(\pi D^{2} \rho U^{2}\right)$, where $F_{D}$ is the drag force on the sphere. The variation of $C_{D}$ for a solid sphere with a Reynolds number of $\operatorname{Re}=\rho D U / \mu$, where $\mu$ is the fluid shear viscosity, is well known. In the subcritical range $5 \times 10^{3}<\operatorname{Re}<3 \times 10^{5}$, the flow around a solid sphere is characterized by flow separation occurring at about the equator of the sphere where the form drag is dominant, giving rise to $C_{D}$ that is approximately constant in the range
$0.44-0.55$. At a critical value of about $\operatorname{Re} \approx 2.5 \times 10^{5}$, the boundary layer becomes turbulent and the flow separation point on the surface moves downstream towards the rear of the sphere. This is accompanied by a dramatic reduction in the drag coefficient to about $C_{D} \approx 0.1-0.2$. This phenomenon is called the drag crisis [7-15].

Drag reduction on bluff bodies moving at high Reynolds numbers $\left(10^{4}-10^{6}\right)$ is commonly achieved by inducing the onset of the drag crisis to occur at lower Re by adding surface features, such as dimples or tripping wires, or by the addition of small amounts of soluble viscoelastic polymers to the fluid phase [8-13]. Recently, we have also shown that the introduction of a sustained vapor layer on the surface of a sphere via the Leidenfrost effect is also a highly efficient drag reduction technique [14,15]. The experiments with a Leidenfrost vapor layer give an upper bound estimate for the lubricating effect of gas layers sustained on superhydrophobic surfaces or induced by microbubble injection [14-20].

In the present study to characterize the drag crisis transition of ice spheres in water, we use ice-shellmetal-core spheres of 40 or $60 \mathrm{~mm}$ outer diameter, produced using a custom-designed aluminimum mold (see Fig. S1 in the Supplemental Material [21]). The core metallic sphere (steel, $\rho=7.8 \mathrm{~g} / \mathrm{cm}^{3}$, or tungsten carbide, $\rho=14.9 \mathrm{~g} / \mathrm{cm}^{3}$ ) is suspended inside the spherical mold and the remaining outer void is filled with water. The mold is then placed inside a temperature chamber to freeze for about $2 \mathrm{~h}$ at $-10^{\circ} \mathrm{C}$ (if not otherwise specified). Details of the production protocol of such ice-covered metal spheres are given in the Supplemental Material [21]. An important aspect of the sphere preparation protocol is that before the release of the ice sphere from the mold, the mold is left to condition at room temperature until the ice surface starts to melt (see Fig. S2 of Supplemental Material [21]). Thus, in all of our experiments the ice-shell temperature is close to 

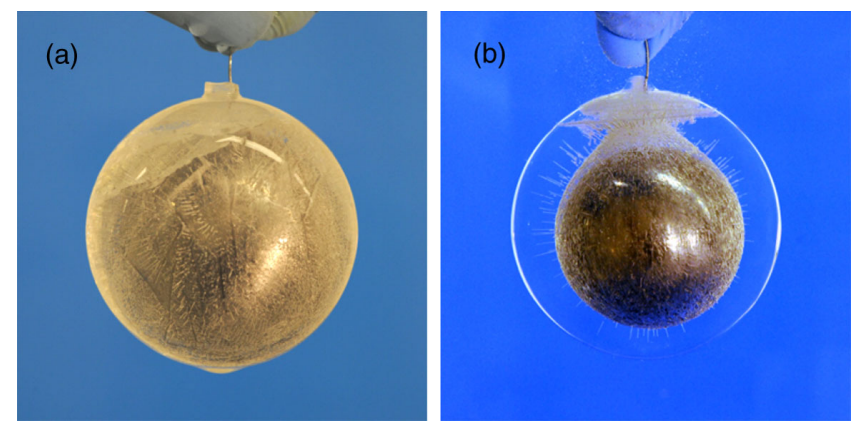

FIG. 1 (color online). Images of a $60 \mathrm{~mm}$ diameter ice-shell sphere with a $40 \mathrm{~mm}$ diameter spherical steel core. (a) Image of the sphere before immersion in water. After the sphere is released from the mold, the ice surface starts to melt slowly and a thin water layer wets the surface, resulting in a glossy appearance. (b) Image of the ice sphere immersed in water. See other examples in Figs. S3 and S8 in the Supplemental Material [21].

zero, and when in air, the ice surface is covered by a thin water film. In Fig. 1, we show a $60 \mathrm{~mm}$ diameter ice sphere with a $40 \mathrm{~mm}$ diameter steel sphere core held in ambient air [Fig. 1(a)] or immersed in water [Fig. 1(b)]. The thin water layer that wets the surface gives a glossy appearance and prevents fog or frost formation on the sphere surface when it is first exposed to the laboratory atmosphere.

Sphere free fall trajectories in water were recorded in a rectangular tank (height $2.4 \mathrm{~m}$, cross section $40 \mathrm{~cm} \times$ $40 \mathrm{~cm}$ ) with a high-speed video camera (Photron SA-5, typical frame rate of 1000 frames/s). In Fig. 2 we compared the free fall velocity of a $60 \mathrm{~mm}$ diameter ice shell with tungsten carbide core and a $60 \mathrm{~mm}$ diameter solid steel sphere of the same weight. The data demonstrate that near the bottom of the tank, the velocity of the ice-covered tungsten carbide sphere is about 1.34 times that of the solid steel sphere, corresponding to drag reduction of about $50 \%$. The data also show that the ice-covered tungsten carbide sphere is still accelerating near the bottom of the $2.4 \mathrm{~m}$ tank. In such cases, an extrapolation function can be used to estimate the terminal velocity $[13,15]$, whereas using the actual velocity measured near the bottom of the tank would give an upper bound estimate of the drag coefficient. Taking the extrapolated terminal velocity value $U_{T}$ gives $C_{D}=0.13$ for the ice sphere, a value that is lower than $C_{D} \sim 0.2$ measured for the solid sphere at the drag crises minimum [11,13].

To investigate the dependence of the drag coefficient $C_{D}$ on the Reynolds number Re, we use 40 and $60 \mathrm{~mm}$ icecovered metal spheres with different size metallic core spheres to span the range $2 \times 10^{4}<\operatorname{Re}<3 \times 10^{5}$. Results for the drag coefficient of the ice-covered spheres and solid metal spheres are shown in Fig. 3. We also used a $60 \mathrm{~mm}$ diameter hollow aluminium sphere that is fitted with a metallic sphere inside, so that its weight matches exactly that of the $60 \mathrm{~mm}$ diameter ice-covered metal sphere to provide a more accurate comparison (see Fig. S4 of

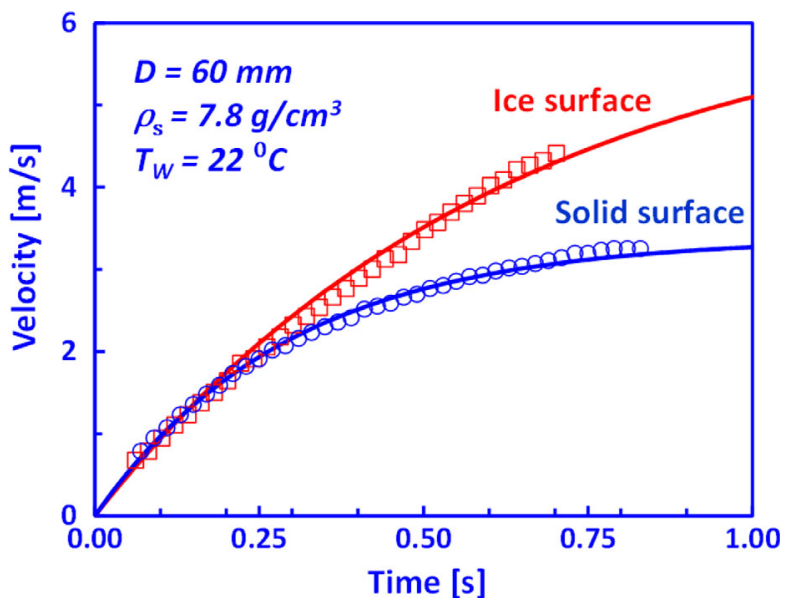

FIG. 2 (color online). Fall velocities versus time for a $60 \mathrm{~mm}$ diameter ice-covered tungsten carbide sphere and a $60 \mathrm{~mm}$ diameter solid steel sphere of the same average density, $\rho_{s}=7.8 \mathrm{~g} / \mathrm{cm}^{3}$, during free fall in room temperature $\left(22^{\circ} \mathrm{C}\right)$ water. Each data series is fitted with the extrapolation function [13] $U(t)=U_{T}\left(1-e^{-t / \tau}\right)$ (lines). An upper bound estimate based on the maximum measured velocity gives $C_{D}=0.23$ for the ice sphere and $C_{D}=0.50$ for the solid sphere. See Video 1 of the Supplemental Material [21].

Supplemental Material [21]). The $C_{D}$ values of ice-covered metal spheres shown in Fig. 3 with solid square symbols are calculated using the sphere velocity measured close to the bottom of the tank, which as discussed above gives an upper bound estimate of the drag coefficient. The data shown as open squares are estimates for the drag coefficients of ice spheres at $\operatorname{Re}>1.5 \times 10^{5}$ calculated using the extrapolated values for the terminal velocity. Use of this extrapolated estimate of the terminal velocity will give a smaller drag coefficient. We note, however, that the extrapolation equation $U(t)=U_{T}\left(1-e^{-t / \tau}\right)$ is an empirical relation proven to give accurate prediction for the terminal velocities of solid spheres falling at Re close to the drag crises, but might not represent correctly the complex dynamics of the true fall trajectories $[13,15]$.

The data in Fig. 3 show that for $\operatorname{Re}<8 \times 10^{4}$ the drag force for the ice-covered metal spheres and the solid sphere are identical, with a typical precrisis value in the range $C_{D}=0.48-0.52$. The drag coefficient for the ice spheres starts to deviate from the precrisis values for $\operatorname{Re}>10^{5}$, and reaches the postdrag crisis values at $\operatorname{Re}=3 \times 10^{5}$. The drag crisis for ice spheres develops over a larger range of Reynolds numbers than in the case of a solid sphere.

The cooling of the water in the boundary layer near the surface of the ice spheres is expected to increase the viscosity locally, possibly giving rise to an increase in the critical Reynolds number. To quantify this effect, we conducted experiments with $60 \mathrm{~mm}$ solid steel spheres that were preconditioned in an environmental chamber to temperatures from $20^{\circ} \mathrm{C}$ down to $-30^{\circ} \mathrm{C}$. For spheres 


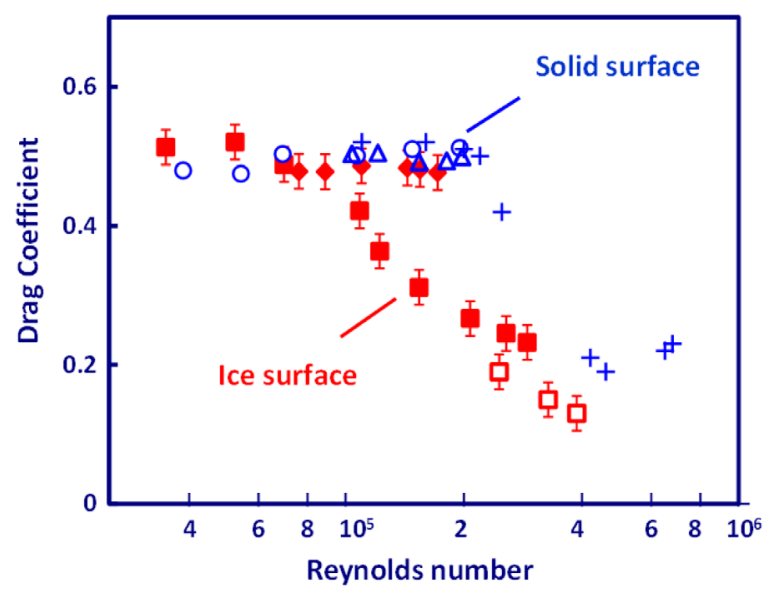

FIG. 3 (color online). Drag coefficient dependence on the Reynolds number for ice surface spheres and smooth solid surface spheres free falling in room temperature $\left(22^{\circ} \mathrm{C}\right)$ water: solid squares (red) are for ice-shell sphere, open circles (blue) are for solid steel spheres, and open triangles (blue) are for solid surface composite spheres matching the weight and the size of the ice-shell spheres [21]. Open squares (red) are drag coefficients for ice-shell spheres calculated using the extrapolated values for the terminal velocity. Solid diamonds (red) are drag coefficients for ice-shell spheres falling in $6 \pm 1{ }^{\circ} \mathrm{C}$ water. Literature values for smooth solid surface spheres falling in an infinite tank measured by White [11] are shown as blue crosses.

conditioned to temperatures below $0{ }^{\circ} \mathrm{C}$, the sphere surface was defrosted before immersion into the water tank by a brief wash with ethanol (the ethanol melting temperature is about $-114^{\circ} \mathrm{C}$, Fig. S5 of Supplemental Material [21]). We found the terminal velocity to be independent of the steel sphere precondition temperature (see Figs. S6 and S7 of the Supplemental Material [21]), thus proving that boundary layer cooling has no measurable effect on the drag reduction herein.

Drag reduction at the onset of the drag crisis is usually associated with the reduction of the form drag due to the offset of the boundary layer separation point from the equator towards the rear of the sphere, often referred to as the delayed separation [7]. In the case of the ice sphere, we confirm this effect by putting a small amount of red dye in the water used in making the ice shell to visualise the wake behind the falling sphere. Snapshots comparing the wake behind a $60 \mathrm{~mm}$ ice-covered metal sphere falling at a velocity corresponding to the Reynolds numbers below $\left(\operatorname{Re} \approx 5 \times 10^{4}\right)$ and above $\left(\operatorname{Re} \approx 1.5 \times 10^{5}\right)$ the critical value are shown in Fig. 4. The entire trajectory is shown in Video 2 of the Supplemental Material [21]. The dye visualization experiment also demonstrates that water from the melting ice surface is continually convected into the trailing wake of the sphere and provides direct evidence that melting of the ice surface is key to understanding the observed drag reduction.

Although the delayed separation demonstrated in the above experiment is the characteristic signature of the drag

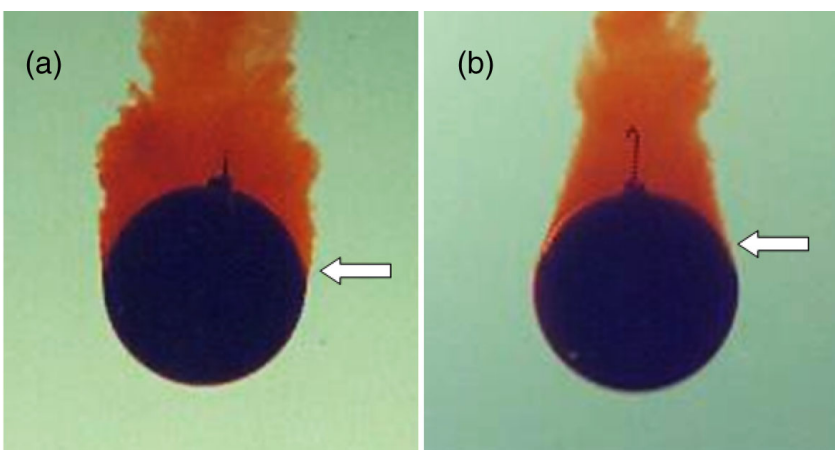

FIG. 4 (color online). High-speed camera snapshots of $60 \mathrm{~mm}$ diameter dyed-ice ice-covered metal sphere falling in water (a) at a low speed of about $0.8 \mathrm{~m} / \mathrm{s}$ corresponding to $\operatorname{Re} \approx 5 \times 10^{4}$ and (b) at a higher speed of about $2.4 \mathrm{~m} / \mathrm{s}$ corresponding to $\operatorname{Re} \approx 1.5 \times 10^{5}$. Arrows indicate the approximate position at which flow separation occurs. See Video 2 of the Supplemental Material [21].

crisis, it can in fact be the result from very different physical mechanisms (see Sec. 6 of the Supplemental Material for a more detailed discussion [21]). Drag reduction by engineered surface features operates by triggering an early transition to the turbulent regime in a boundary layer, resulting in a delayed separation [7-9]. In contrast, the addition of viscoelastic polymer near the surface introduces a local non-Newtonian response that promotes the delayed separation $[11,12]$. Drag reduction can also result from the creation of a low density and low viscosity vapor layer on the surface that changes the no-slip boundary condition at the solid-liquid interface to one of partial or full-slip boundary condition at the vapor-liquid interface that in turn delays the boundary layer separation [14-19].

In the present case of melting surfaces we consider two possible physical mechanisms that can contribute to the early onset of drag reduction: (i) the change from the noslip to partial-slip boundary condition at the water-ice surface, or (ii) the disturbance of the boundary layer due to the constant mass transfer of water from the melting of the ice surface. To quantify these hypotheses we conducted a series of experiments to measure the characteristic melting rate of the falling spheres in terms of the rate of change of the sphere diameter $|d D / d t|$ (see Sec. 4 and Fig. S8 of the Supplemental Material [21]). A quantitative comparison of the slip and mass transfer hypotheses based on measurement of the characteristic melting rate is given in Sec. 6 of the Supplemental Material [21]. In brief, from the measured surface melting rate, $|d D / d t| \sim 0.6 \pm$ $0.2 \mathrm{~mm} / \mathrm{s}$, for a $60 \mathrm{~mm}$ sphere that falls with velocity $U_{T} \sim$ $2 \mathrm{~m} / \mathrm{s}\left(\operatorname{Re} \sim 1.2 \times 10^{5}\right)$, we estimated the ratio between the effective slip length $\lambda_{S}$ at the ice surface and the thickness of the viscous boundary layer $\lambda_{B}$ to be $\lambda_{S} / \lambda_{B} \sim$ $0.5|d D / d t| /\left(U_{T}\right) \sim 0.0015$. These estimates show that the effective slip is about 3 orders of magnitude lower than the boundary layer thickness and is an unlikely source 
of the drag reduction observed. On the other hand, to estimate how the mass transfer due to surface melting can affect the boundary layer thickness, we consider the characteristic melting thickness defined as the thickness change of the ice layer as the sphere moves over a distance of one diameter $D[21]$ :

$$
\lambda_{M} \sim 0.5|d D / d t|\left(D / U_{T}\right) .
$$

Taking $|d D / d t| \sim 0.6 \mathrm{~mm} / \mathrm{s}, D=60 \mathrm{~mm}$, and $U_{T} \sim 2 \mathrm{~m} / \mathrm{s}$, we estimate the characteristic melting thickness scale, $\lambda_{M} \sim 10 \mu \mathrm{m}$, which is less than but still a significant fraction of the characteristic thickness of the boundary layer $\lambda_{B} \sim D / \operatorname{Re}^{1 / 2} \sim 170 \mu \mathrm{m}$, at the same sphere size and Reynolds number [22,23]. Although $\lambda_{M}$ is estimated to be only $5 \%-10 \%$ of thickness of the boundary layer on a solid sphere, we believe that this could be enough to trigger the disturbance of the viscous boundary layer that lead to the early onset of the drag crisis observed in our experiment.

The above analysis indicates that the key factor that initiated the early onset of the drag crisis in the case of a melting surface is the rate of the mass transfer into the boundary layer and is physically very different from the case of a vapor layer where the dominant effect is the change to a slip boundary condition at the vapor-liquid interface. Development of a more quantitative model of boundary layer and drag on bluff bodies integrating mass transfer and slip effect remains a formidable theoretical and computational challenge.

Further support of the notion that the drag reduction is related to the melting rate of the ice surface was obtained with measurement of ice-covered metal spheres falling in $6 \pm 1{ }^{\circ} \mathrm{C}$ water prepared by mixing the room temperature water in the tank with an appropriate amount of ice cubes [21]. Under such conditions, the ice melting rate was 20 times lower than that for room temperature $\left(22 \pm 1{ }^{\circ} \mathrm{C}\right)$ water [21]. The results presented in Fig. 3 (solid diamonds) show no detectable differences between the drag coefficient of ice spheres falling in $6^{\circ} \mathrm{C}$ water and a solid sphere for the experimentally accessible range of $\operatorname{Re}$ of up to $1.7 \times 10^{5}$. A side-by-side comparison (see Video 3 of the Supplemental Material [21]) of an ice-shell sphere and a solid sphere of identical size and mean density in $6{ }^{\circ} \mathrm{C}$ water clearly demonstrates that both spheres fall at the same rate at $\operatorname{Re}=1.5 \times 10^{5}$, whereas the ice sphere falls much faster in $22{ }^{\circ} \mathrm{C}$ water (Video 1 of Supplemental Material [21]). These results confirm the hypotheses that melting of the ice surface is responsible for the observed drag reduction effect.

To our knowledge, the effect of a melting solid-liquid interface has not been considered as a factor that can affect the separation of the boundary viscous layer and more generally the hydrodynamic properties of bluff bodies moving in liquid. A detailed understanding of the mechanics of the ice melting drag reduction could lead to means of reproducing the effect in other systems involving solid phase melting or dissolution in the surrounding liquid phase.

Research reported in this publication is supported by the King Abdullah University of Science and Technology (KAUST). We also acknowledge the KAUST Machine Workshop and Mr. Tayyab Mubeen for the production of the ice molds. This work is supported in part by an Australian Research Council Discovery Project Grant to DYCC.

*ivanuriev.vakarelski@kaust.edu.sa

†D.Chan@unimelb.edu.au

[1] R. Rosenberg, Phys. Today 58, No. 12, 50 (2005).

[2] J. G. Dash, H. Fu, and J. S. Wettlaufer, Rep. Prog. Phys. 58, 115 (1995).

[3] A. Doppenschmidt and H.-J. Butt, Langmuir 16, 6709 (2000).

[4] Y. Li and G. A. Somorjai, J. Phys. Chem. C 111, 9631 (2007).

[5] A.-M. Kietzig, S. G. Hatzikiriakos, and P. Englezos, J. Appl. Phys. 107, 081101 (2010).

[6] M. P. Langleben, J. Geophys. Res. 87, 573 (1982).

[7] E. Achenbach, J. Fluid Mech. 54, 565 (1972).

[8] J. Choi, W-P. Jeon, and H. Choi, Phys. Fluids 18, 041702 (2006).

[9] H. Choi, W-P. Jeon, and J. Kim, Annu. Rev. Fluid Mech. 40, 113 (2008).

[10] B. Dean and B. Bhushan, Phil. Trans. R. Soc. A 368, 4775 (2010).

[11] A. White, Nature (London) 216, 994 (1967).

[12] C. M. White and M. G. Mungal, Annu. Rev. Fluid Mech. 40, 235 (2008).

[13] N. Lyotard, W. L. Shew, L. Bocquet, and J.-F. Pinton, Eur. Phys. J. B 60, 469 (2007).

[14] I. U. Vakarelski, J. O. Marston, D. Y. C. Chan, and S. T. Thoroddsen, Phys. Rev. Lett. 106, 214501 (2011).

[15] I. U. Vakarelski, D. Y. C. Chan, and S. T. Thoroddsen, Soft Matter 10, 5662 (2014).

[16] C. Cottin-Bizonne, J.-L. Barrat, L. Bocquet, and E. Charlaix, Nat. Mater. 2, 237 (2003).

[17] J. P. Rothstein, Annu. Rev. Fluid Mech. 42, 89 (2010).

[18] S. L. Ceccio, Annu. Rev. Fluid Mech. 42, 183 (2010).

[19] G. McHale, M. I. Newton, and N. J. Shirtcliffe, Soft Matter 6, 714 (2010).

[20] I. U. Vakarelski, N. A. Patankar, J. O. Marston, D. Y. C. Chan, and S. T. Thoroddsen, Nature (London) 489, 274 (2012).

[21] See Supplemental Material at http://link.aps.org/ supplemental/10.1103/PhysRevLett.115.044501 for experimental details, supplemental figures (Figs. S1-S8), and video clips (Videos 1-3).

[22] H. Schlichting, Boundary Layer Theory (McGraw-Hill Book Company, New York, 1979).

[23] S. Tomotika, Technical Report of the Aeronautical Research Committee for the Year 1935-1936, R. \& M. No. 1678, 86-99 (1937). 